\title{
Highly Luminescent Gels and Mesogens based on
}

\section{Elaborated Borondipyrromethenes}

\author{
By Franck Camerel, Laure Bonardi, Marc Schmutz and Raymond Ziessel
}

\section{Supporting information (5 pages)}

\section{Experimental Section}

General Methods. The $300.1\left({ }^{1} \mathrm{H}\right)$ and $75.5 \mathrm{MHz}\left({ }^{13} \mathrm{C}\right)$ and $128.3 \mathrm{MHz}\left({ }^{11} \mathrm{~B}\right) \mathrm{NMR}$ spectra were recorded at room temperature using perdeuterated solvents as internal standards: \%o $(\mathrm{H})$ in ppm relative to residual protiated solvent; \%o (C) in ppm relative to the solvent. The $128.3 \mathrm{MHz}\left({ }^{11} \mathrm{~B}\right) \mathrm{NMR}$ spectra were recorded at variable temperature using glass residual $\mathrm{B}_{2} \mathrm{O}_{3}$ as internal standards $\mathrm{A}$ fast-atom bombardment ZAB-HF-VB-analytical apparatus in positive mode was used with a $m$-nitrobenzyl alcohol ( $m$-NBA) as matrix. FT-IR spectra were recorded on the neat liquids or as thin films, prepared with a drop of dichloromethane, and evaporated to dryness on $\mathrm{KBr}$ pellets. Temperature dependent FTIR measurements were performed on gels in IR cells with $\mathrm{KBr}$ windows or on solids in anhydrous $\mathrm{KBr}$ pellets with a home-made heating stage (room temperature up to $250{ }^{\circ} \mathrm{C}$ ). UV-vis spectra were recorded using a UVIKON 940/941 dual-beam grating spectrophotometer (Kontron Instruments) with a $1 \mathrm{~cm}$ quartz cell. Fluorescence spectra were recorded on a Perkin-Elmer LS50B spectrofluorimeter. Fluorescence temperature-dependence measurements on gels were performed in $2 \mathrm{~mm}$ inner diameter quartz capillary. The temperature was controlled with a $0.5 \mathrm{~mm}$ diameter thermocouple directly inserted in the gel. The heating stage was constructed with a flexible heating filament wrapped around the capillary support and connected to a standard electronic control unit. Differential Scanning Calorimetry (DSC) was performed on a Netzsch DSC 200 PC/1/M/H Phox ${ }^{\circledR}$ instrument equipped with an intracooler, allowing measurements from $-65^{\circ} \mathrm{C}$ up to $450{ }^{\circ} \mathrm{C}$. The samples were examined at a scanning rate of 10 K.min ${ }^{-1}$ by applying two heating and one cooling cycles. The apparatus was calibrated with indium 
$\left(156.6^{\circ} \mathrm{C}\right)$. Phase behavior was studied by polarized light optical microscopy (POM) on a Leica DMLB microscope equipped with a Linkam LTS350 hot-stage and a Linkam TMS94 central processor. Fluorescence temperature-dependence microscopy was performed with a Leica DMLB fluorescence microscope equipped with a continuous $100 \mathrm{~W}$ mercury lamp and with a Linkam LTS350 hot-stage and a Linkam TMS94 central processor. Transmission electron microscopy (TEM) experiments were performed with a CM12 Philips microscope on diluted solution deposited onto a carbon coated grid and on metallic replicas of the gels obtained by the freeze fracture technique.

Chromatographic purification was conducted using 40-63 $\mu \mathrm{m}$ silica gel. Thin layer chromatography (TLC) was performed on silica gel plates coated with fluorescent indicator. All mixtures of solvents are given in $v / v$ ratio. The experimental procedures for each reaction were tested several times to optimally find the best conditions.

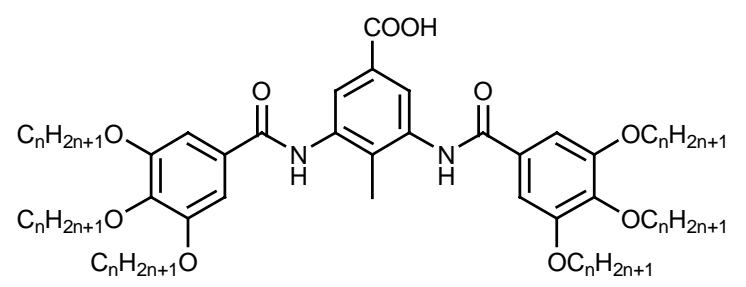

A; $n=16$

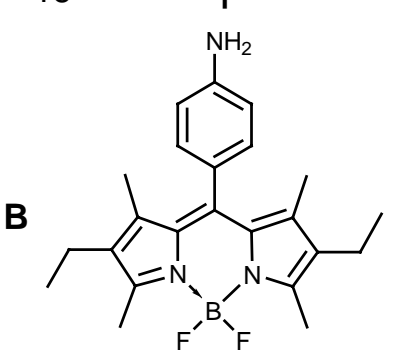

(i)<smiles></smiles>

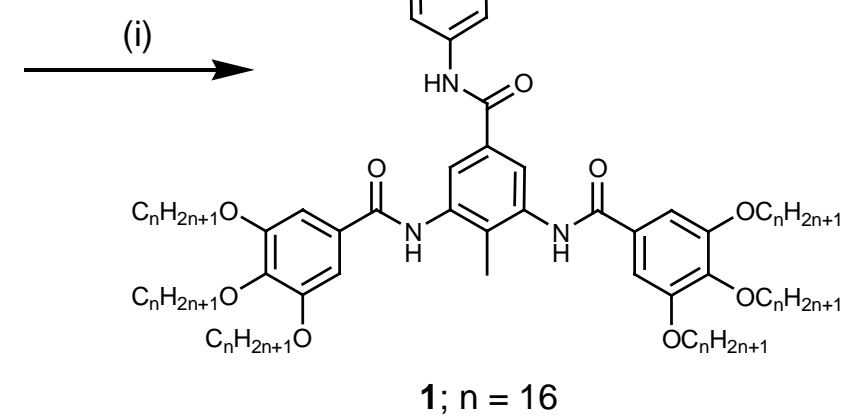

Materials. Compounds A with $\mathrm{n}=16$ were synthesized according to reference $\mathrm{S} 1$ and compound $\mathbf{B}$ according to reference $\mathrm{S} 2$.

Compound 1. In a round-bottomed flask, to a stirred solution of $\mathbf{B}$ (1.2 equiv, $0.050 \mathrm{~g}, 0.13 \mathrm{mmol}$ ) in distilled $\mathrm{CH}_{2} \mathrm{Cl}_{2}(50 \mathrm{~mL})$, were added sequentially A (1 equiv, $0.10 \mathrm{mmol}, 0.192 \mathrm{~g}$ ), 1-ethyl-3-(3dimethylaminopropyl)-carbodiimide (2 equiv, $0.21 \mathrm{mmol}, 0.040 \mathrm{~g}$ ), and 2,2-dimethylaminopyridine (2 
equiv, $0.21 \mathrm{mmol}, 0.026 \mathrm{~g}$ ). The reaction mixture was heated until complete solubilization of the starting material and then stirred at room temperature until TLC $\left(\mathrm{SiO}_{2} ; \mathrm{CH}_{2} \mathrm{Cl}_{2} / \mathrm{MeOH}\right.$ 99:1) indicated no evolution of the reaction (about 48 hours). The reaction mixture was washed with water $(3 \times 15 \mathrm{~mL})$ and extracted with $\mathrm{CH}_{2} \mathrm{Cl}_{2}(20 \mathrm{~mL})$. The organic layer was dried over $\mathrm{MgSO}_{4}$, filtered and the solvent evaporated. The crude product was purified by chromatography on a column packed with silica gel, using $\mathrm{CH}_{2} \mathrm{Cl}_{2} / \mathrm{MeOH}$ (100:0 to 99:1) as eluent. Recrystallization in $\mathrm{CH}_{2} \mathrm{Cl}_{2} / \mathrm{CH}_{3} \mathrm{CN}$ mixtures afforded the desired compound as a red powder $(0.172 \mathrm{~g}, 74 \%)$.

${ }^{1} \mathrm{H} \mathrm{NMR}\left(\mathrm{CDCl}_{3}, 300 \mathrm{MHz}\right): \delta(\mathrm{ppm})=8.76(\mathrm{~s}, \mathrm{br}, 1 \mathrm{H}), 8.10(\mathrm{~s}, \mathrm{br}, 2 \mathrm{H}), 7.96(\mathrm{~s}, 2 \mathrm{H}), 7.52(\mathrm{AB}, 4 \mathrm{H}$, $\left.{ }^{\mathrm{AB}} J=8.5 \mathrm{~Hz}, v \delta_{\mathrm{AB}}=378 \mathrm{~Hz}\right), 7.15(\mathrm{~s}, 4 \mathrm{H}), 4.01(\mathrm{~m}, 12 \mathrm{H}), 2.52(\mathrm{~s}, 6 \mathrm{H}), 2.28\left(\mathrm{q}, 4 \mathrm{H},{ }^{3} J=7.3 \mathrm{~Hz}\right), 2.18$ (s, 3H), 1.88-1.69 (m, 12H), 1.55-1.18 (m, 162H), $0.96\left(\mathrm{t}, 6 \mathrm{H},{ }^{3} \mathrm{~J}=7.3 \mathrm{~Hz}\right), 0.91-0.82(\mathrm{~m}, 18 \mathrm{H}) ;{ }^{13} \mathrm{C}$ $\operatorname{NMR}\left(\mathrm{CDCl}_{3}, 75 \mathrm{MHz}\right): \delta(\mathrm{ppm})=166.3,153.8,153.5,142.1,139.8,138.9,138.5,136.9,132.9,131.8$, $131.1,129.1,128.7,121.6,120.7,106.2,73.8,69.6,32.1,30.5,29.9,29.8,29.64,29.57,29.5,26.31$, 26.28, 17.2, 14.8, 14.3, 13.5, 12.6, $12.0 ;{ }^{11} \mathrm{~B}$ NMR $\left(\mathrm{CDCl}_{3}, 128 \mathrm{MHz}\right): \delta(\mathrm{ppm})=3.86\left(\mathrm{t},{ }^{1} J=32 \mathrm{~Hz}\right)$; UV-Vis $\left(\mathrm{CH}_{2} \mathrm{Cl}_{2}\right) \lambda(\mathrm{nm})\left(\varepsilon, \mathrm{M}^{-1} \mathrm{~cm}^{-1}\right)=525$ (70000), 495 (sh, 23000), 367 (9000), 276 (54000), 229 (67000) ; IR $\left(\mathrm{KBr}, \mathrm{cm}^{-1}\right): v=3281,2918,2850,1645,1582,1538,1464,1332,1188,1114,979$; $\mathrm{FAB}^{+}-\mathrm{MS} m / z$ (nature of peak, relative intensity) : 2193.2 ([M+H] $\left.]^{+}, 100\right), 2173.1$ ([M-F], 15) ; Anal. Calcd for $\mathrm{C}_{141} \mathrm{H}_{236} \mathrm{BF}_{2} \mathrm{~N}_{5} \mathrm{O}_{9}: \mathrm{C}, 77.18 ; \mathrm{H}, 10.84 ; \mathrm{N}, 3.19$. Found : C, $76.82 ; \mathrm{H}, 10.67 ; \mathrm{N}, 2.91$.

\section{References}

S1. Pickaert, G.; Cesario, M.; Ziessel, R. J. Org. Chem. 2004, 69, 5335.

S2. Azov, V.A.; Skinner, P.J.; Yamakoshi, Y.; Seiler, P.; Gramlich, V.; Diederich, F. Helvetica Chimica Acta 2003, 86, 3648. 


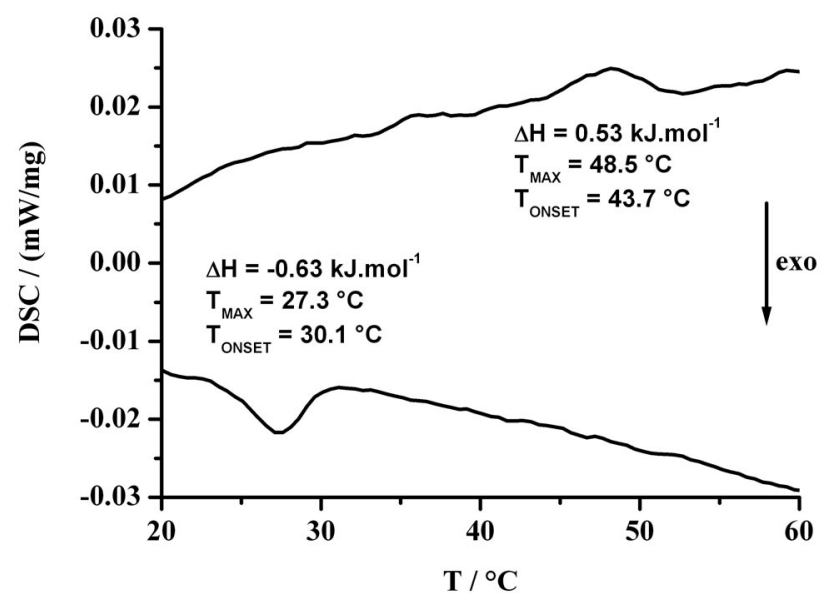

Figure S1. DSC Traces of a gel of compound 1 in nonane (22 g.L $\left.\mathrm{L}^{-1}\right)$ (top: second heating curve; bottom: first cooling curve). 

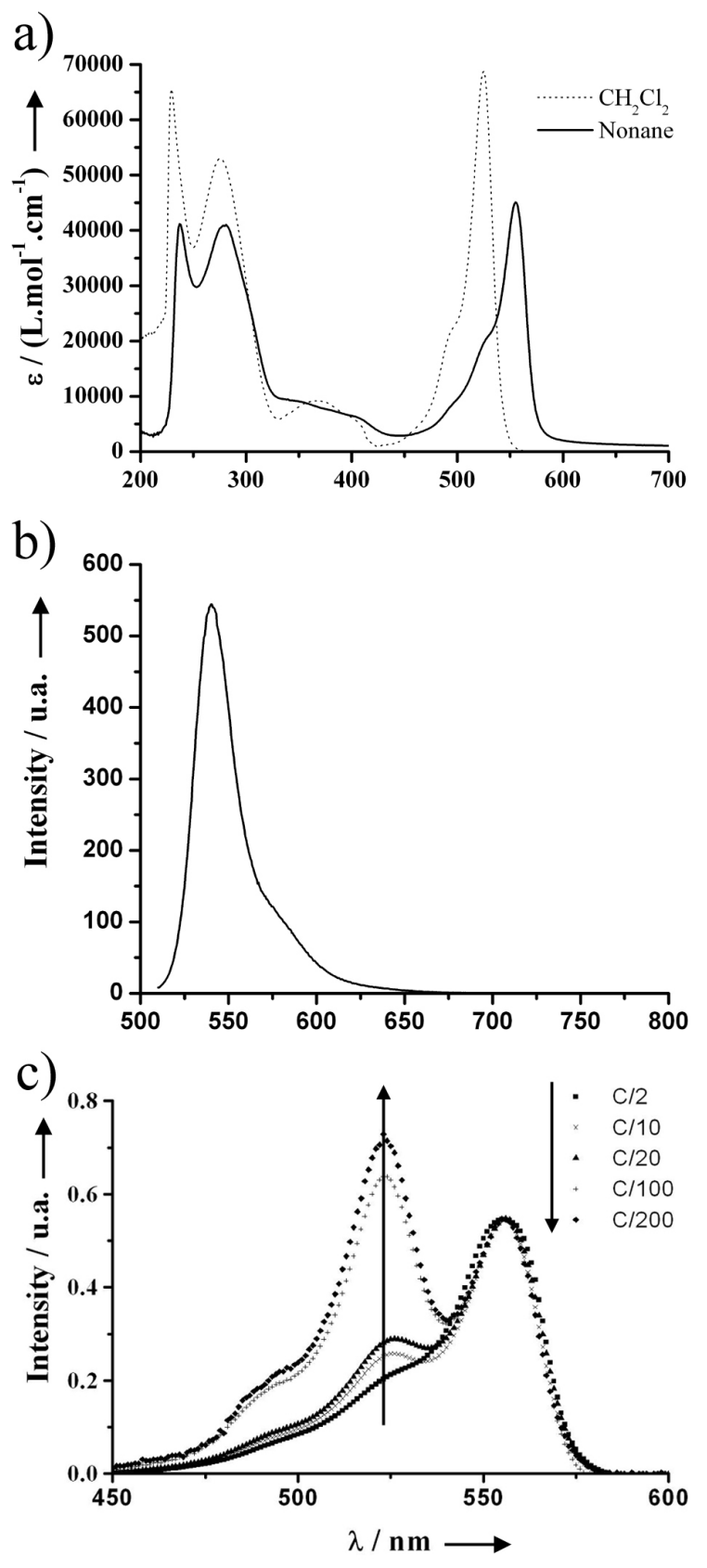

Figure S2. a) Absorption spectra of compound 1 in dichloromethane $\left(\mathrm{C}=1.5 \times 10^{-5} \mathrm{~mol} . \mathrm{L}^{-1}\right)$ and in nonane $\left(\mathrm{C}=3.6 \times 10^{-5} \mathrm{~mol} . \mathrm{L}^{-1}\right)$; b) Emission spectrum of compound $\mathbf{1}$ in $\mathrm{CH}_{2} \mathrm{Cl}_{2}(\mathrm{C}=$ $\left.\left.9.6 \times 10^{-7} \mathrm{~mol} . \mathrm{L}^{-1}\right)\left(\lambda_{\mathrm{ex}}=490 \mathrm{~nm}\right) ; \mathrm{c}\right)$ Normalized absorption spectra at $555 \mathrm{~nm}$ of compound 1 in nonane at different concentrations $\left(\mathrm{C}=10^{-3} \mathrm{~mol} \cdot \mathrm{L}^{-1}\right)$. 